\title{
CIRCLES OF NUMBERS
}

\author{
by M. BURMESTER, R. FORCADE and E. JACOBS
}

(Received 12 November, 1976)

Introduction. Arrange any $n$ integers around a circle. The following procedure can be used to obtain another circle of $n$ integers. For each adjacent pair of the first integers, form the absolute value of their difference and place it between them; then remove the original numbers. This procedure can be repeated over and over. When $n=4$ this always leads eventually to a circle of zeros. On the other hand when $n=3$, unless the original numbers are equal, this never happens. We treat below the general case and related problems, using for convenience a slightly different formulation. Surprisingly there is enough structure to lead to some interesting mathematics.

DefinITIONS. Let $C=\left(x_{i}\right)_{i=1}^{\infty}$ be an infinite sequence of numbers. Let $\Psi(C)=\left(x_{i}^{\prime}\right)_{i=1}^{\infty}$ where $x_{i}^{\prime}=\left|x_{i+1}-x_{i}\right|$ for all $i$. We call $C$ an $n$-cycle if and only if $x_{i+n}=x_{i}$ for all $i$. We shall also represent an $n$-cycle as an $n$-tuple $\left(x_{1}, x_{2}, \ldots, x_{n}\right)$ when it is convenient.

REMARKS.

1. If $C$ is an $n$-cycle so is $\Psi(C)$.

2 . Any $n$-cycle is also a $k n$-cycle ( $k$ any natural number).

3. After one application of $\Psi$ all $x_{i}$ 's are non-negative.

4. Let $m(C)=\max _{i}\left(x_{i}\right)$ (which exists for $n$-cycles). Then $m(\Psi(C)) \leq m(C)$ if all entries in $C$ are non-negative.

Definition. $C$ will be called repeating if and only if $\Psi^{k}(C)=C$ for some $k$. The smallest such $k$ will be called the period of $C$.

5 . The 0 -cycle $(0,0, \ldots, 0)$ is the only repeating cycle with period 1 .

6. In view of Remark 4 , every $n$-cycle made up of integers (or rational numbers) must eventually, under repeated application of $\Psi$, be reduced to a repeating one. This is not always true for cycles made up of irrational numbers (for example, if $C=$ $\left(1, r, r^{2}, \ldots, r^{n}\right)$ where $r^{n}=1+r+r^{2}+\ldots+r^{n-1}$, then $\left.\Psi^{t}(C)=(r-1)^{t} C, t=1,2, \ldots\right)$.

7. Observe that $\Psi\left(F_{n}, F_{n+1}, F_{n+2}\right)=\left(F_{n-1}, F_{n}, F_{n+1}\right)$, where $F_{n}$ is the $n$-th Fibonacci number. The 3-cycle $\left(F_{n}, F_{n+1}, F_{n+2}\right)$ requires $n$ applications of $\Psi$ to reach a repeating cycle. Note also that $F_{n}=5^{-\frac{1}{2}}\left(t^{n}-s^{n}\right)$ where $t$ and $s$ are respectively the positive and negative roots of $x^{2}=x+1$ (see Remark 6).

Discussion AND DEFINTIIONs. We are concerned primarily with $n$-cycles made up of integers, and with what happens to such cycles after repeated application of $\Psi$. In view of Remark 3 we may as well assume that all entries are non-negative (so Remark 4 applies). We are interested in knowing which cycles lead finally to the 0 -cycle. These will be called terminating cycles, and all others will be called non-terminating.

Definition. A cycle made up only of 0's and 1's will be called a primitive cycle.

Glasgow Math. J. 19 (1978) 115-119 
THEOREM 1. Every repeating $n$-cycle is a constant multiple of a primitive $n$-cycle.

Proof. By Remark 4, it is clear that $m(C)=m\left(\Psi^{t}(C)\right), t=1,2, \ldots$, for any repeating cycle $C$. Thus $C$ is made up entirely of non-negative numbers $\leq m$, where $m=m(C)$. Furthermore there must be at least one occurrence of $m$ in $C$. Now let $C_{1}$ be $C$ 's unique repeating preimage under $\Psi\left(C_{1}=\Psi^{k-1}(C)\right.$, where $k$ is the period of $\left.C\right)$. Since $m\left(C_{1}\right)=m$, the two entries of $C_{1}$ whose absolute difference is the entry $m$ of $C$ must be $0, m$ (or $m, 0$ ). Similarly if $C_{2}$ is the repeating preimage of $C_{1}$, the three entries of $C_{2}$ whose absolute difference give us $0, m$ (or $m, 0$ ) must be taken from the set $\{0, m\}$. If we continue working backwards in this way through the repeating preimages of $C$, we get that the $n$-th preimage $C_{n}$ of $C$ has a string of $n$ successive entries all taken from $\{0, m\}$ (with $m$ occurring at least once). Since $C_{n}$ is an $n$-cycle it has to be a constant multiple of a primitive cycle. The proof is completed by observing that $C=\Psi^{n}\left(C_{n}\right)$.

THEOREM 2. Let $C$ be a primitive $n$-cycle, $n=2^{k} L$ where $L$ is odd.

(i) $C$ is terminating if and only if it is a $2^{k}$-cycle.

(ii) $C$ is repeating if and only if it (as an $n$-tuple vector over $\mathbf{Z}_{2}$ ) is orthogonal to every primitive terminating $n$-cycle.

Proof. Observe that if $C=\left(x_{i}\right)_{i=1}^{\infty}$ then $\left|x_{i+1}-x_{i}\right| \equiv x_{i+1}+x_{i}(\bmod 2)$. Thus $\Psi$ becomes a linear operator on the $n$-dimensional vector space (over $\mathbf{Z}_{2}$ ) of primitive $n$-cycles. Also notice that $\Psi=I+E$ where $E\left(x_{1}, x_{2}, \ldots\right)=\left(x_{2}, x_{3}, \ldots\right)$. Thus $\Psi^{r}=(I+E)^{r}=\sum_{j=0}^{r}\left(\begin{array}{l}r \\ j\end{array}\right) E^{j}$ and therefore $\Psi^{\prime}(C)=\left(y_{r i}\right)_{i=1}^{\infty}$ where $y_{r i}=\sum_{j=0}^{r}\left(\begin{array}{l}r \\ j\end{array}\right) x_{i+j}$ (addition being over $\mathbf{Z}_{2}$ ). So $C$ is terminating if and only if $\sum_{j=0}^{r}\left(\begin{array}{l}r \\ j\end{array}\right) x_{i+j}=0$ for some $r$ and all $i$. We may assume, without loss of generality, that $r$ is a power of 2 , in which case $\left(\begin{array}{l}r \\ 0\end{array}\right)$ and $\left(\begin{array}{l}r \\ r\end{array}\right)$ are odd and all other $\left(\begin{array}{l}r \\ j\end{array}\right)$ are even ( $\equiv 0$ in $\mathbf{Z}_{2}$ ). Thus $C$ is terminating if and only if $x_{i}=x_{i+r}$ for some $r$ (a power of 2) and for all $i$. This is equivalent to $C$ being a $r$-cycle which, since $C$ is already a $2^{k} L$-cycle, is equivalent to $C$ being a $2^{k}$-cycle.

For the second part of the theorem notice that $E(u) . v=u . E^{-1}(v)$ (for any vectors $u$ and $v$ ), so the fact that $E$ leaves invariant the subspace $T$ of primitive $n$-cycles implies that $E$ leaves invariant its orthogonal complement $R$ (all $n$-cycles orthogonal to $T$ ). Thus $\Psi=I+E$ must also preserve $R$. But $T$ already contains the kernel of $\Psi$ so $\Psi$ must be a nonsingular linear transformation when restricted to $R$. Thus $R$ must consist entirely of repeating $n$-cycles. Since $R$ is the orthogonal complement of $T$ any element not in $R$ is representable (uniquely) as the sum of a non-zero vector of $T$ and an element of $R$. Since this sum under repeated application of $\Psi$ is reduced to an element of $R$, all repeating (primitive) $n$-cycles lie in $R$ and the proof is finished.

Observe that in the proof of Theorem 2 we have also established that any primitive $n$-cycle can be uniquely represented as the sum of a repeating and a terminating $n$-cycle. 
THEOREM 3. (i) If $n$ is a power of 2 then every rational $n$-cycle is terminating.

(ii) If $n$ is odd then the only terminating rational $n$-cycles are the trivial ones (those with all entries equal).

(iii) If $n$ is neither a power of 2 nor odd, there are non-terminating rational $n$-cycles as well as non-trivial terminating rational $n$-cycles.

Proof. Every rational $n$-cycle will eventually be reduced to a repeating one (Remark 6) which must be a constant multiple of a primitive cycle by Theorem 1 . If $n$ is a power of 2 then (by Theorem 2) every primitive $n$-cycle is terminating, hence so is every $n$-cycle. If $n$ is odd the only primitive terminating $n$-cycles (by Theorem 2$)$ are $(m, m, \ldots, m)$ where $m=0$ or $m=1$. A simple parity argument shows that $(m, m, \ldots m)$ has no $\Psi$-preimage for any $m>0$. If $n$ is neither a power of 2 nor odd then $n=2^{k} L$ where $k \geq 1$ and $L$ is an odd number greater than 1 . Then there must be terminating $2^{k}$-cycles (which are $n$-cycles by Remark 2 ) and non-terminating $L$-cycles (which are $n$-cycles).

DEFINITION AND DISCUSSION. If $n=2$ it is easy to see that each $n$-cycle terminates in two $\Psi$-steps. One might conjecture that the terminating $n$-cycles which (by Theorem 3 ) exist for every even $n>2$ are all as trivial (vanishing in only a few $\Psi$-steps). That is not the case. Let the life-span of an $n$-cycle be the minimum number of $\Psi$-steps required to make it a repeating cycle. It turns out that both the terminating and non-terminating $n$-cycles whose existence is predicted by Theorem 3 can be specified to have an arbitrary long life-span in every case (except for $n=2$ ). These remarks are an easy consequence of the following theorem.

THEOREM 4. Let $C$ be an $n$-cycle $(n>2)$. Then there exists another $n$-cycle $C_{1}$ and $k>0$ such that $k, \Psi(C)=\Psi^{2}\left(C_{1}\right)$.

Note that $C_{1}$ has a longer life-span than $C$ but that they are of the same type (either terminating or non-terminating).

Before proving Theorem 4 we shall need a few lemmas and definitions.

Lemma 1. Let $C=\left(x_{1}, x_{2}, \ldots, x_{n}\right)$ be an $n$-cycle. Then $C$ has a $\Psi$-preimage if and only if there exists $A \subseteq\{1,2, \ldots, n\}$ for which $\sum_{i \in A} x_{i}=\sum_{i \in A} x_{i}$.

Proof. If $C=\Psi\left(y_{1}, y_{2}, \ldots, y_{n}\right)$ let $A=\left\{i \mid x_{i}=y_{i}-y_{i+1}\right\}$. Then

$$
\sum_{i \in A} x_{i}=\sum_{i \in A}\left(y_{i}-y_{i+1}\right) \text { and } \sum_{i \in A} x_{i}=\sum_{i \notin A}\left(y_{i+1}-y_{i}\right)
$$

So

$$
\sum_{i \in A} x_{i}-\sum_{i \notin A} x_{i}=\sum_{i=1}^{n}\left(y_{i}-y_{i+1}\right)=0
$$

since $y_{n+1}=y_{1}$. Conversely, if there exists such a set $A$, let $y_{1}=\sum_{i \in A} x_{i}$, and $y_{i+1}=y_{i}+\delta_{i} x_{i}$ where $\delta_{i}=1$ if $i \in A$ and $\delta_{i}=-1$ if $i \notin A$. Then $C=\Psi\left(y_{1}, y_{2}, \ldots, y_{n}\right)$. 
DEFINITION. If $C=\left(x_{1}, x_{2}, \ldots, x_{n}\right)$ is an $n$-cycle, a weak predecessor of $C$ is an $n$-cycle $C_{1}$ for which $\Psi\left(C_{1}\right)=\left(a x_{1}+b, a x_{2}+b, \ldots, a x_{n}+b\right)$, where $a, b$ are integers, $a>0$ (Note that in this case $C, C_{1}$ satisfy the equation of Theorem 4).

LeMMA 2. Let $C=\left(x_{1}, x_{2}, \ldots, x_{n}\right), n>2$. Rearrange the $x$ 's into a non-increasing list $y_{1} \geq y_{2} \geq \ldots \geq y_{n}$ and assume that $y_{n}=0$. Then $C$ has a weak predecessor if and only if $\sum_{i=1}^{s} y_{i} \geq \sum_{i=s+1}^{n} y_{i}$, where $s=[(n-1) / 2]$.

Proof. If $C$ has a weak predecessor then by Lemma 1 there exists $A \subseteq\{1,2, \ldots, n\}$, $a>0$ and $b$ such that

$$
\sum_{i \in A}\left(a y_{i}+b\right)=\sum_{i \notin A}\left(a y_{i}+b\right) .
$$

Note that $b \geq 0$ because $y_{n}=0$ and all $a y_{i}+b$ are non-negative by Remark 3 . We can assume without loss of generality that $|A| \leq n-|A|$ (or replace $A$ by its complement in $\{1,2, \ldots, n\})$. Let $t=|A|$ and let $B=\{1,2, \ldots, t\}$. Then

$$
\sum_{i \in B}\left(a y_{i}+b\right) \geq \sum_{i \in A}\left(a y_{i}+b\right)=\sum_{i \notin A}\left(a y_{i}+b\right) \geq \sum_{i \notin B}\left(a y_{i}+b\right) .
$$

Thus $a\left(\sum_{i \in B} y_{i}\right)+b t \geq a\left(\sum_{i \notin B} y_{i}\right)+b(n-t)$, and since $t \leq n-t$ and $b \geq 0$ and $a>0, \sum_{i \in B} y_{i} \geq$ $\sum_{i \in B} y_{i}$. If $t<n / 2$, by taking $s=[(n-1) / 2]$ we certainly get $\sum_{i=1}^{s} y_{i} \geq \sum_{i=s+1}^{n} y_{i}$. If $t=n / 2$ equation (1) reduces to $\sum_{i \in A} y_{i}=\sum_{i \in A} y_{i}$. But since $y_{n}=0$ we may by dropping $n$ from either $A$ or its complement choose a new $A$ with $t-1$ elements for which this equation still holds, and then proceed as before with $a=1, b=0$.

Conversely, if the above equation holds, let $a=n-2 s, b=\sum_{i=1}^{s} y_{i}-\sum_{i=s+1}^{n} y_{i}$. Then $a y_{i}+b \geq 0$ for all $i$ and $\sum_{i=1}^{s}\left(a y_{i}+b\right)=\sum_{i=s+1}^{n}\left(a y_{i}+b\right)$. By Lemma 1 it follows that $C$ has a weak predecessor. We now restate Theorem 4 in the following way.

THEOREM 4A. If $n$ is odd every $n$-cycle has a weak predecessor. If $n$ is even and greater than 2 and $C=\left(x_{1}, x_{2}, \ldots, x_{n}\right)$ is an $n$-cycle, then either $C$ has a weak predecessor or $C^{\prime}=\left(m-x_{1}, m-x_{2}, \ldots, m-x_{n}\right)$ does (here $\left.m=m(C)\right)$.

Proof: If $t=\min _{i}\left(x_{i}\right)$ then $C=\left(x_{1}, x_{2}, \ldots, x_{n}\right)$ has a weak predecessor if and only if $\left(x_{1}-t, x_{2}-t, \ldots, x_{n}-t\right)$ does. Thus the added assumption that $y_{n}=0$ of Lemma 2 applies. But clearly the inequality in Lemma 2 always holds when $n$ is odd (compare the two sums involving the same number of elements, element by element). When $n$ is even the inequality may sometimes fail to hold. If so consider $C^{\prime}$ (which will have the same 
$\Psi$-image as $C)$, so that in effect we replace the $y_{i}$ by $y_{i}^{\prime}=y_{1}-y_{n-i+1}$. Then, letting $n=2 k$, we get

$$
\begin{aligned}
\sum_{i=1}^{k-1} y_{i}^{\prime}-\sum_{i=k}^{n} y_{i}^{\prime} & =-y_{1}+\sum_{i=2}^{k+1} y_{i}-\sum_{i=k+2}^{n-1} y_{i} \\
& =-y_{1}+y_{k}+y_{k+1}+\sum_{i=2}^{k-1} y_{i}-\sum_{i=k+2}^{n-1} y_{i} \\
& \geq-y_{1}+y_{k}+y_{k+1}+\sum_{i=k+2}^{n-1} y_{i}-\sum_{i=2}^{k-1} y_{i} \\
& =\sum_{i=k}^{n-1} y_{i}-\sum_{i=1}^{k-1} y_{i},
\end{aligned}
$$

which is $>0$ by the assumption that $C$ does not have a weak predecessor. Thus we have shown that $C^{\prime}$ does have a weak predecessor.

If $n$ is neither odd nor a power of 2, the problem of distinguishing between terminating and non-terminating $n$-cycles seems quite difficult. It is clear, for example, that an $n$-cycle which is congruent $\bmod 2$ to a repeating primitive $n$-cycle must be non-terminating. But the converse is untrue as shown by the following 6-cycle: $(18,25$, $34,19,0,13)$. Almost any kind of alteration in the $n$-cycle seems capable of changing it from terminating to non-terminating. For example $(89,140,83,0,45,56)$ is terminating, but $(89,142,83,0,45,56)$ is non-terminating. Similarly $(1,4,9,6,5,2)$ is terminating, but with the elements permuted $(1,6,9,2,5,4)$ is non-terminating.

The question of determining the life-span of an $n$-cycle also seems interesting but there appears to be no easy way of tackling it.

Finally, an interesting area to explore is that of $n$-cycles made up of real but possibly irrational numbers. For instance, for some $n$ 's the cycle mentioned in Remark 6 and similar ones seem to be the only cycles having infinite life-span.

Department of MATHEMAtics

Royal Holloway College

EGHAM

SURREY

1208 MAJOR

NORMAL

ILLINOIS

Department of Mathematics

ILLINOIS STATE UNIVERSITY

Normal

ILLINOIS 